IUHET-518

\title{
Higher order Josephson effects
}

\author{
Roman V. Buniy ${ }^{1, *}$ and Thomas W. Kephart ${ }^{2,+}$ \\ ${ }^{1}$ Physics Department, Indiana University, Bloomington, IN 47405 \\ ${ }^{2}$ Department of Physics and Astronomy, \\ Vanderbilt University, Nashville, TN 37235
}

(Dated: August 13, 2008)

\begin{abstract}
Gaussian linking of superconducting loops containing Josephson junctions with enclosed magnetic fields give rise to interference shifts in the phase that modulates the current carried through the loop, proportional to the magnitude of the enclosed flux. We generalize these results to higher order linking of a superconducting loop with several magnetic solenoids, and show there may be interference shifts proportional to the product of two or more fluxes.
\end{abstract}

*Electronic address: roman.buniy@gmail.com

$\dagger$ Electronic address: tom.kephart@gmail.com 


\section{INTRODUCTION}

Interference effects, both constructive and destructive, are mainstays for distinguishing between quantum and classical phenomena. Examples include interfering scattering amplitudes, both bosonic and fermionic, formation of condensates, entanglement, etc. The Aharonov-Bohm effect [1], describes the self-interference of a charged particle that can travel along two semiclassical paths whose combined path is gaussian linked with a magnetic solenoid carrying flux $\Phi$. The measurable phase shift is $\phi \propto \Phi$. We have argued in Ref. [2] that there could exist generalizations to cases of higher order linkings. The simplest example is a Borromean ring arrangement where the semiclassical path corresponds to one ring, which has higher order linking with two flux tubes carrying fluxes $\Phi_{1}$ and $\Phi_{2}$, which make up the other two rings. We found the phase shift in this system is $\phi \propto \Phi_{1} \Phi_{2}$. Higher order cases were explored in Ref. [3, 4] and shown to be related to commutator algebras of homotopy generators of the configuration space $\mathbb{R}^{3} \backslash\left\{T_{1} \cup T_{2}\right\}$, where $T_{1}$ and $T_{2}$ are the tubes containing the fluxes. The same general logic can be applied to systems of superconductors,

Josephson junctions, and magnetic fluxes where the Josephson effect can arise [5]. Here we will study interference in a symmetric arrangement of two identical semicircular superconductors joined by two identical Josephson junctions and derive the response of such systems. We conclude with a discussion of possible applications.

In the case of gaussian linking of a loop of superconductor with a magnetic solenoid, the Mercereau effect [6] is due to the phase change in the macroscopic wave function, which is in turn related to the currents in the superconducting components. The effect is due to the presence of a vector potential $\boldsymbol{A}$, which is the fundamental object responsible for the phase change. Exploration of higher order linking is again due to the presence of a vector potential but in these instances it requires careful choices of gauge.

\section{THE JOSEPHSON EFFECT}

It will be sufficient for our purposes to consider a macroscopic model of superconductors. Following Feynman [7], we approximate the superconductors coupled via a Josephson junction as a two-level system. Let $\psi_{1}$ and $\psi_{2}$ be the states, $E_{1}$ and $E_{2}$ the energy levels of the superconductors. The Schrodinger equation for the coupled system of the two 
superconductors becomes

$$
\begin{aligned}
& i \hbar\left(\partial \psi_{1} / \partial t\right)=E_{1} \psi_{1}+K \exp (i \phi) \psi_{2}, \\
& i \hbar\left(\partial \psi_{2} / \partial t\right)=E_{2} \psi_{2}+K \exp (-i \phi) \psi_{1}
\end{aligned}
$$

where $K$ is the coupling energy and $\phi$ is a phase, which arises from the most general hermitian hamiltonian $2 \times 2$ matrix. The dependence of $\phi$ on the vector potential $\boldsymbol{A}$ can be found from gauge invariance considerations. For a gauge transformation $\boldsymbol{A} \mapsto \boldsymbol{A}+\boldsymbol{\nabla} f, \psi_{1} \mapsto$ $\psi_{1} \exp (i q f / 2 \hbar c), \psi_{2} \mapsto \psi_{2} \exp (-i q f / 2 \hbar c)$ with an arbitrary function $f$ of space coordinates, we find $\phi \mapsto \phi+q f / \hbar c$, from which it follows that $\phi=(q / \hbar c) \int \boldsymbol{A} \cdot d \boldsymbol{x}$. Here $q=2 e$ is the charge of an electron pair.

After the substitutions $\psi_{1}=\left|\psi_{1}\right| \exp \left(i \theta_{1}\right)$ and $\psi_{2}=\left|\psi_{2}\right| \exp \left(i \theta_{2}\right)$, the Schrodinger equation becomes

$$
\begin{aligned}
\hbar\left(\partial\left|\psi_{1}\right|^{2} / \partial t\right) & =2 K\left|\psi_{1}\right|\left|\psi_{2}\right| \sin \theta, \\
\hbar\left(\partial\left|\psi_{2}\right|^{2} / \partial t\right) & =-2 K\left|\psi_{1}\right|\left|\psi_{2}\right| \sin \theta, \\
-\hbar\left|\psi_{1}\right|\left(\partial \theta_{1} / \partial t\right) & =E_{1}\left|\psi_{1}\right|+K\left|\psi_{2}\right| \cos \theta, \\
-\hbar\left|\psi_{2}\right|\left(\partial \theta_{2} / \partial t\right) & =E_{2}\left|\psi_{2}\right|+K\left|\psi_{1}\right| \cos \theta,
\end{aligned}
$$

where $\theta=\phi+\theta_{2}-\theta_{1}$. The current from superconductor 1 to superconductor 2, which is equal to minus the current from superconductor 2 to superconductor 1 , is thus $I=(2 K / \hbar)\left|\psi_{1}\right|\left|\psi_{2}\right| \sin \theta$. (In a self-consistent computation, a current from a battery which connects the two superconductors is also included. The result for the superconducting current is precisely I; see, for example, Ref. [8].) The electron densities in the two superconductors are approximately equal and independent of time; let $\rho$ be this common constant. This gives $I=I_{0} \sin \theta$, where $I_{0}=2 K \rho / \hbar$. Integrating the phase equations, we find

$$
\theta(t)=\phi+\theta(0)+\hbar^{-1} \int_{0}^{t} d t^{\prime}\left(E_{1}\left(t^{\prime}\right)-E_{2}\left(t^{\prime}\right)\right) .
$$

The quantity $\left(E_{1}(t)-E_{2}(t)\right) / q$ represents an electric potential applied to the junction. The de and ac Josephson effects [5] arise for $\left|E_{1}(t)-E_{2}(t)\right| \ll K$ and $\left|E_{1}(t)-E_{2}(t)\right| \gg K$, respectively.

Our interest here is in the Josephson effect with zero potential across the junction, $E_{1}(t)-$ $E_{2}(t)=0$, and nonzero magnetic field constrained to the opening of the superconducting 


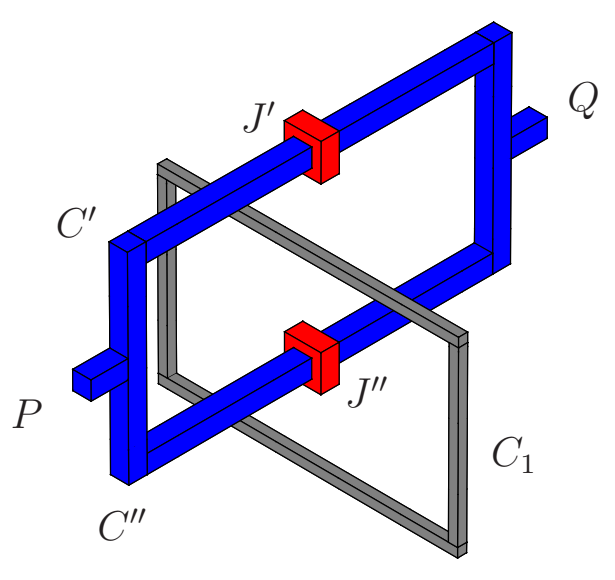

FIG. 1: A diagram of an experimental setup for the detection of the Josephson effect. $C^{\prime}$ and $C^{\prime \prime}$ are paths from the point $P$ to the point $Q$ through the superconductors with the Josephson junctions $J^{\prime}$ and $J^{\prime \prime}$ and the total current $I$ from $P$ to $Q . C_{1}$ is the magnetic solenoid carrying flux $\Phi_{1}$. The Josephson effect (for a review see Ref. [9]) is due to the first order (gaussian) linking of the closed curves $C=C^{\prime} C^{\prime \prime-1}$ and $C_{1}$.

ring with two Josephson junctions; see Fig. 1, Let $\theta^{\prime}$ and $\theta^{\prime \prime}$ be the phase changes due to the vector potential $\boldsymbol{A}_{1}$ of the currents through the junctions $J^{\prime}$ and $J^{\prime \prime}$. The phase changes from the point $P$ to the point $Q$ along the paths $C^{\prime}$ and $C^{\prime \prime}$ are

$$
\begin{aligned}
& \phi^{\prime}=\theta^{\prime}+(q / \hbar c) \int_{C^{\prime}} \boldsymbol{A}_{1} \cdot d \boldsymbol{x}, \\
& \phi^{\prime \prime}=\theta^{\prime \prime}+(q / \hbar c) \int_{C^{\prime \prime}} \boldsymbol{A}_{1} \cdot d \boldsymbol{x} .
\end{aligned}
$$

Since the wave function is single valued, this requires $\phi^{\prime}=\phi^{\prime \prime}$, and so we find $\theta^{\prime \prime}-\theta^{\prime}=$ $2 \pi \Phi_{1} / \Phi_{0}$. Here $\Phi_{1}=\oint_{C} \boldsymbol{A}_{1} \cdot d \boldsymbol{x}$ is the flux due to the solenoid along $C_{1}$ passing through a surface spanned by a closed curve $C=C^{\prime} C^{\prime \prime-1}$ and $\Phi_{0}=2 \pi \hbar c / q$ is the flux quantum. The total current from the point $P$ to the point $Q$ is

$$
I=I_{0} \sin \left(\frac{1}{2}\left(\theta^{\prime}+\theta^{\prime \prime}\right)\right) \cos \left(\pi \Phi_{1} / \Phi_{0}\right)
$$

For a fixed value of $\Phi_{1}$, the corresponding maximal total current is

$$
I_{\max }=I_{0}\left|\cos \left(\pi \Phi_{1} / \Phi_{0}\right)\right|
$$

which itself has maxima when $\Phi_{1}=n \Phi_{0}, n \in \mathbb{Z}$.

The flux is actually $\Phi_{1}=\Phi_{1 \text {,ext }}+L I$ where $\Phi_{1 \text {,ext }}$ is the external flux through the loop, $L$ is the self-inductance, but here and in what follows we assume $L$ is negligible. (We have 
made a number of simplifying assumptions, for example, that self-inductance of SQUID components are negligible, none of which, if relaxed, affect our basic conclusions.)

We will call the phenomena reviewed in this section first order Josephson effects to distinguish them from their generalizations which we now proceed to describe.

\section{THE SECOND AND HIGHER ORDER JOSEPHSON EFFECTS}

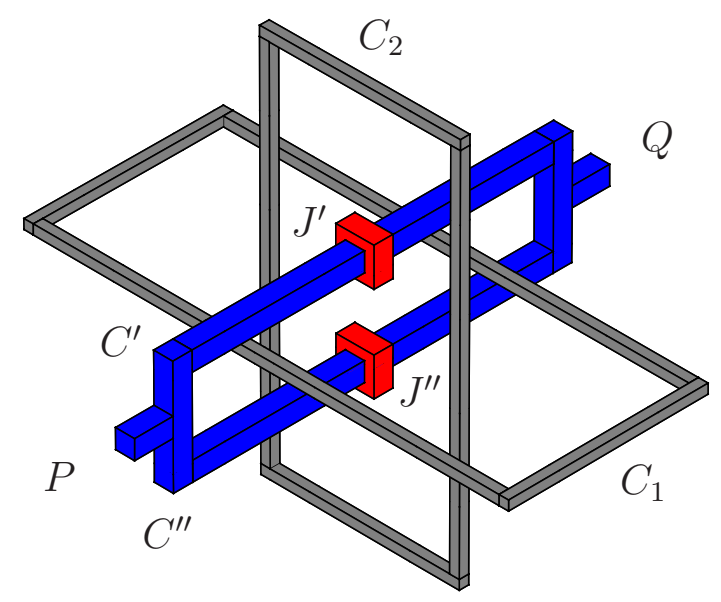

FIG. 2: A diagram of an experimental setup for the detection of the second order Josephson effect. $C_{1}$ and $C_{2}$ are the magnetic solenoids. $C^{\prime}$ and $C^{\prime \prime}$ are paths from the point $P$ to the point $Q$ through the superconductors connected by Josephson junctions $J^{\prime}$ and $J^{\prime \prime}$. The total current from $P$ to $Q$ is $I . C_{1}$ and $C_{2}$ are magnetic solenoids carrying fluxes $\Phi_{1}$ and $\Phi_{2}$. The second order Josephson effect is due to the second order linking of the set of three closed curves $C=C^{\prime} C^{\prime \prime-1}$, $C_{1}$ and $C_{2}$.

Now consider the case where we have two solenoids carrying magnetic fluxes $\Phi_{1}$ and $\Phi_{2}$ and whose center lines run along $C_{1}$ and $C_{2}$, and a superconducting ring along the closed curve $C=C^{\prime} C^{\prime \prime-1}$ with two Josephson junctions $J^{\prime}$ and $J^{\prime \prime}$ in parallel as shown in Fig. 2. The two solenoids and the superconducting ring are in a Borromean rings [10] configuration. Note that in this arrangement neither $C_{1}$ nor $C_{2}$ has gaussian linking with the superconducting ring $C$, nor do $C_{1}$ and $C_{2}$ link with each other. However, the set of three $\operatorname{rings} C, C_{1}, C_{2}$ is indeed linked. This second order linking and its higher order generalizations is what will lead to our results. In other words, we will find that even though our system lacks first order (gaussian) linking, a phase difference can still exist upon traveling around the 
superconductor. To find this phase, we must choose a gauge that detects it. Such a gauge is [2] $\boldsymbol{A}_{12}=\frac{1}{2} k_{2}\left(\gamma_{1} \boldsymbol{A}_{2}-\gamma_{2} \boldsymbol{A}_{1}\right)$. Here $\boldsymbol{A}_{1}$ and $\boldsymbol{A}_{2}$ are the vector potentials due to the solenoids along $C_{1}$ and $C_{2}$, the quantities $\gamma_{1}$ and $\gamma_{2}$ are defined by

$$
\gamma_{j}=\delta_{j}+(q / \hbar c) \int_{\Gamma} \boldsymbol{A}_{j} \cdot d \boldsymbol{x}
$$

where $\delta_{1}$ and $\delta_{2}$ are constants, and $\Gamma$ is a path that runs along $C$. The quantity $k_{2}$ is a normalization constant, the value of which we discuss below.

Using $\boldsymbol{A}_{12}$ in the phase integral and following computations in Ref. [2], we find $\theta^{\prime \prime}-\theta^{\prime}=$ $4 \pi^{2} k_{2} \Phi_{1} \Phi_{2} / \Phi_{0}^{2}$. The total current from point $P$ to point $Q$ is

$$
I=I_{0} \sin \left(\frac{1}{2}\left(\theta^{\prime}+\theta^{\prime \prime}\right)\right) \cos \left(2 \pi^{2} k_{2} \Phi_{1} \Phi_{2} / \Phi_{0}^{2}\right) .
$$

For fixed values of $\Phi_{1}$ and $\Phi_{2}$, the maximal total current flowing in the superconductor is

$$
I_{\max }=I_{0}\left|\cos \left(2 \pi^{2} k_{2} \Phi_{1} \Phi_{2} / \Phi_{0}^{2}\right)\right| .
$$

The smallest value of the constant $k_{2}>0$ for which the fluxes $\Phi_{1}=m_{1} \Phi_{0}, \Phi_{2}=m_{2} \Phi_{0}$, where $m_{1}, m_{2} \in \mathbb{Z}$, lead to maxima of the quantity $I_{\max }$ is $k_{2}=(2 \pi)^{-1}$. This is precisely the value we obtained in Ref. [2] by imposing an analog of the Dirac string condition on the second order phase for the Aharonov-Bohm effect. Nevertheless, the value of $k_{2}$ must ultimately be determined by experiment.

Also, for the value $k_{2}=(2 \pi)^{-1}$, if either $\Phi_{1}$ or $\Phi_{2}$ is equal to $\Phi_{0}$ or $-\Phi_{0}$, then in terms of the other flux, appropriately relabeled, the expression (14) for the second order $I_{\max }$ reduces to the expression (11) for the first order $I_{\max }$.

An essential feature of the above result is that the current $I$ is a periodic function of the quantity $\pi k_{2} \Phi_{1} \Phi_{2} / \Phi_{0}^{2}$ with period 1 . We can also derive this property by modifying the method used by Block [11] for the first order Josephson effect as follows.

The total gauge potential includes internal and external parts, $\boldsymbol{A}=\boldsymbol{A}_{\text {in }}+\boldsymbol{A}_{\text {ext }}$, the external magnetic field being due to the external sources. Assuming that the external field $\boldsymbol{\nabla} \times \boldsymbol{A}_{\text {ext }}$ vanishes inside the superconductors, we can write $\boldsymbol{A}_{\text {ext }}=\boldsymbol{\nabla} \gamma_{\text {ext }}$. As a result, $\boldsymbol{A}$ is a gauge transformation of $\boldsymbol{A}_{\text {in }}$, and so

$$
\psi\left(\boldsymbol{A}_{\text {in }}+\boldsymbol{A}_{\text {ext }}\right)=\psi\left(\boldsymbol{A}_{\text {in }}\right) \exp \left(i q \gamma_{\text {ext }} / \hbar c\right) .
$$

Since $\psi(\boldsymbol{A})$ is single valued, we find that $\psi\left(\boldsymbol{A}_{\text {in }}\right)$ is multiplied by the factor $\exp \left(-4 i \pi^{2} k_{2} \Phi_{1} \Phi_{2} / \Phi_{0}^{2}\right)$ after the charge $q$ travels around a closed curve $C$. This factor is a 
periodic function of $\pi k_{2} \Phi_{1} \Phi_{2} / \Phi_{0}^{2}$ with period 2 . This implies the same periodicity property for the wave function $\psi\left(\boldsymbol{A}_{\text {in }}\right)$ and the energy $E$. Assuming time reversal symmetry as in Ref. [11], we find that the free energy and thus the current, which is given by minus the derivative of the free energy with respect to the external flux, are both periodic functions of $\pi k_{2} \Phi_{1} \Phi_{2} / \Phi_{0}^{2}$ with period 1 , in agreement with the result proved earlier.

More generally, it is straightforward to arrange $n$ solenoids with the fluxes $\Phi_{1}, \ldots, \Phi_{n}$ and a superconducting ring in such a way that they are linked with nonzero $n$th order linking [10]. We similarly find the phase difference

$$
\theta^{\prime \prime}-\theta^{\prime}=(2 \pi)^{n} k_{n} \Phi_{1} \cdots \Phi_{n} / \Phi_{0}^{n},
$$

the current

$$
I=I_{0} \sin \left(\frac{1}{2}\left(\theta^{\prime}+\theta^{\prime \prime}\right)\right) \cos \left(\frac{1}{2}(2 \pi)^{n} k_{n} \Phi_{1} \cdots \Phi_{n} / \Phi_{0}^{n}\right),
$$

and its maximal value for fixed values of $\Phi_{1}, \ldots, \Phi_{n}$,

$$
I_{\max }=I_{0}\left|\cos \left(\frac{1}{2}(2 \pi)^{n} k_{n} \Phi_{1} \cdots \Phi_{n} / \Phi_{0}^{n}\right)\right| .
$$

Other properties of these systems can be investigated.

The smallest value of the constant $k_{n}>0$ for which the fluxes $\Phi_{j}=m_{j} \Phi_{0}$, where $m_{j} \in \mathbb{Z}$, lead to maxima of the quantity $I_{\max }$ is $k_{n}=(2 \pi)^{1-n}$. Again this is precisely the value we obtained in Refs. [2, 3] by imposing an analog of the Dirac string condition on the phase for the $n$th order Aharonov-Bohm effect. Nevertheless, as pointed out with the $k_{2}$ case above, the value of $k_{n}$ must ultimately be determined by experiment.

Also, for the value $k_{n}=(2 \pi)^{1-n}$, if one of the fluxes is equal to $\Phi_{0}$ or $-\Phi_{0}$, then in terms of the remaining fluxes, appropriately relabeled, the expression (18) for the $n$th order $I_{\max }$ reduces to the analogous expression (18) for the $(n-1)$ st order $I_{\max }$.

Similar to the case $n=2$ above, we can modifying the method used by Block and prove that for any $n$ the current $I$ is a periodic function of the quantity $\frac{1}{2}(2 \pi)^{n-1} k_{n} \Phi_{1} \cdots \Phi_{n} / \Phi_{0}^{n}$ with period 1.

\section{CONCLUSION}

We generalize the Josephson effect to its higher order analogs in which a superconducting loop links with several magnetic solenoids and the resulting interference shifts are proportional to the product of two or more fluxes. 
One can conceive of a number of applications for devices build to take advantage of higher order linking. Such a system could be less invasive than first order devices because it could keep the SQUID some distance from an experimental sample. Possible applications include both rf and dc SQUIDs that measure higher order linking of multiple fluxes. Under some circumstances such devices could be useful in measurements of complex biological systems, or any systems where direct gaussian linking of a magnetic flux with a SQUID is impractical, but where higher order linking is possible. For example, one could have a system of (i) a fixed but adjustable flux tube, i.e., a solenoid; (ii) an unknown flux to be measured, and (iii) a SQUID. If the three components can be arranged to have higher-order linking, then the unknown flux could be measured, even though it has no gaussian linking with the SQUID.

\section{Acknowledgments}

The work of RVB was supported by DOE grant number DE-FG02-91ER40661 and that of TWK by DOE grant number DE-FG05-85ER40226.

[1] Y. Aharonov and D. Bohm, Phys. Rev. 115, 485 (1959).

[2] R. V. Buniy and T. W. Kephart, Phys. Lett. A 372, 2583 (2008) arXiv:hep-th/0611334.

[3] R. V. Buniy and T. W. Kephart, Phys. Lett. A 372, 4775 (2008) arXiv:hep-th/0611335.

[4] R. V. Buniy and T. W. Kephart, arXiv:hep-th/0611336.

[5] B. D. Josephson, Physics Letters, 1, 251 (1962).

[6] R. C. Jaklevic, J. Lambe, A. H. Silver, and J. E. Mercereau, Phys. Rev. Lett. 12, 159 (1964), Phys. Rev. Lett. 12, 274 (1964).

[7] R. P. Feynman, R. B. Leighton and M. Sands, Feynman Lectures on Physics, Addison-Wesley, Reading, MA, 1965, volume III, chapter 21.

[8] H. A. Ohta, IC-SQUID 76, 35 (1976).

[9] A. A. Golubov, M. Yu. Kupriyanov, and E. Il'ichev Rev. Mod. Phys. 76, 411 (2004)

[10] D. Rolfson, Knots and Links, Publish or Perish, Wilmington, DE (1976).

[11] F. Block, Phys. Rev. B, 2, 109 (1970). 